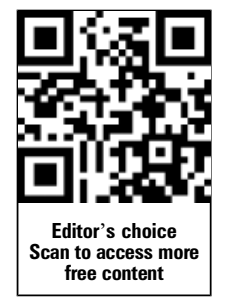

${ }^{1}$ Centre for Exercise and Sports Science, School of Exercise and Nutrition Sciences, Deakin University, Geelong, Victoria, Australia

2Department of Physiology, Australian Institute of Sport, Canberra, Australian Capital Territory, Australia

${ }^{3}$ Department of Movement Science, Biomechanics, Australian Institute of Sport, Canberra, Australian Capital Territory, Australia ${ }^{4}$ Centre for Physical Activity and Nutrition Research, School of Exercise and Nutrition Sciences, Deakin University, Melbourne, Victoria, Australia ${ }^{5}$ Division of Physiotherapy, The University of Queensland, Brisbane, Queensland, Australia

\section{Correspondence to} Dr Jason Bonacci, Centre for Exercise and Sports Science, School of Exercise and Nutrition Sciences, Deakin University, Geelong, VIC 3216, Australia; jason.bonacci@deakin.edu.au

Received 27 September 2012 Revised 15 November 2012 Accepted 17 December 2012 Published Online First 11 January 2013

\title{
Running in a minimalist and lightweight shoe is not the same as running barefoot: a biomechanical study
}

\author{
Jason Bonacci, ${ }^{1}$ Philo U Saunders, ${ }^{2}$ Amy Hicks, ${ }^{3}$ Timo Rantalainen, ${ }^{4}$ \\ Bill (Guglielmo) T Vicenzino, ${ }^{5}$ Wayne Spratford ${ }^{3}$
}

\section{ABSTRACT}

Aim The purpose of this study was to determine the changes in running mechanics that occur when highly trained runners run barefoot and in a minimalist shoe, and specifically if running in a minimalist shoe replicates barefoot running.

Methods Ground reaction force data and kinematics were collected from 22 highly trained runners during overground running while barefoot and in three shod conditions (minimalist shoe, racing flat and the athlete's regular shoe). Three-dimensional net joint moments and subsequent net powers and work were computed using Newton-Euler inverse dynamics. Joint kinematic and kinetic variables were statistically compared between barefoot and shod conditions using a multivariate analysis of variance for repeated measures and standardised mean differences calculated.

Results There were significant differences between barefoot and shod conditions for kinematic and kinetic variables at the knee and ankle, with no differences between shod conditions. Barefoot running demonstrated less knee flexion during midstance, an $11 \%$ decrease in the peak internal knee extension and abduction moments and a $24 \%$ decrease in negative work done at the knee compared with shod conditions. The ankle demonstrated less dorsiflexion at initial contact, a $14 \%$ increase in peak power generation and a $19 \%$ increase in the positive work done during barefoot running compared with shod conditions. Conclusions Barefoot running was different to all shod conditions. Barefoot running changes the amount of work done at the knee and ankle joints and this may have therapeutic and performance implications for runners.

\section{INTRODUCTION}

Most modern running shoes typically feature heavily cushioned and elevated heels, thick midsoles, arch supports and motion control features. Despite unsubstantiated claims about the benefits of barefoot running for running-related injury prevention, ${ }^{1}$ the majority of competitive runners wear shoes. An alternative proposition has emerged where manufacturers have developed 'minimalist' running shoes, which have a lower profile, greater sole flexibility, reduced heel-forefoot offset and lack motion control and the heavy cushioning features of conventional running shoes. Despite the plethora of minimalist shoes on the market there is little evidence to support the notion that the mechanics of running in a minimalist shoe is different to a conventional running shoe and/or similar to barefoot running.

Several studies have compared the dynamics of barefoot running with that of shod running. ${ }^{2-6}$ The most consistent findings are a reduction in stride length, a less dorsiflexed ankle at initial contact and a shift from a rearfoot to midfoot or forefoot strike when barefoot compared with shod. Other findings include a reduction in knee flexion excursion ${ }^{7}$ and knee joint moments ${ }^{6}$ during stance when running barefoot compared with shod running. These studies give insight into the mechanical differences between running in a conventional running shoe and barefoot, but provide no information regarding the mechanics of running in minimalist shoes. Only one study has compared the mechanics of barefoot and minimalist shoe running, but it only studied sagittal plane kinematics in a small cohort of eight runners on a treadmill. ${ }^{8}$ That study found similar ankle contact angles between barefoot and minimalist shoes but kinematics and kinetics of the ankle during treadmill running are not entirely representative of overground running. ${ }^{9}{ }^{10}$ Moreover, alterations in frontal and transverse plane joint motion have been associated with overuse running injuries, ${ }^{11-13}$ and that study did not investigate joint kinetics which can explain the biomechanical cause of observed changes in joint movement patterns. In view of these limitations and the recent interest in these minimalist designs, further research is needed to better understand the mechanical effects of barefoot and minimalist shoe running. The purpose of this study was twofold: (1) to determine whether the mechanics of running in a minimalist shoe are similar to running barefoot in habitually shod runners and (1) to examine changes in lower limb running kinematics and kinetics that occur when habitually shod highly trained runners run barefoot and in a minimalist shoe. We hypothesised that: (1) minimalist shoes would replicate the dynamics of barefoot running and (2) barefoot running would induce changes in lower limb running kinematics and kinetics of highly trained runners.

\section{METHODS}

\section{Participants}

Twenty-two (14 men and 8 women) highly trained runners were recruited for the study. The participants had a mean (SD) age of 29.2 (6.0) years; height of $1.76(0.07) \mathrm{m}$ and body mass of 65.6 $(8.8) \mathrm{kg}$. All participants were training for competition at the time of testing (see table 1 for details) and their mean (SD) personal best $10 \mathrm{~km}$ time in 
Table 1 Mean (SD) participant training history and distance run during the familiarisation period

\begin{tabular}{lc}
\hline & Mean (SD) \\
\hline Training history & \\
km per session & $13.6(3.7)$ \\
Sessions per week & $7.6(2.0)$ \\
km per week & $105.3(33.5)$ \\
Familiarisation period $(\mathrm{km})$ & \\
Barefoot & $4.2(2.1)$ \\
Minimalist & $24.3(16.4)$ \\
Racing flat & $22.2(13.7)$ \\
Regular & $78.0(42.7)$ \\
\hline
\end{tabular}

the previous 12 months was $33 \mathrm{~min} 41 \mathrm{~s} \quad(3 \mathrm{~min} \quad 43 \mathrm{~s}$ ). Participants were excluded from the study if they had suffered from any musculoskeletal or neurological condition that prevented them from training in the previous 3 months. Written informed consent was obtained from all participants and procedures were approved by the Deakin University and Australian Institute of Sport Human Ethics Committees.

\section{Experimental conditions}

The protocol involved four experimental conditions: (1) barefoot; (2) a minimalist shoe (NIKE Free 3.0); (3) a lightweight racing flat (NIKE LunaRacer2) and (4) the shoe in which they were currently completing the most training mileage (herein called regular shoe). The NIKE Free 3.0 fits the category of minimalist as it has an ultraflexible sole, is lightweight, has a reduced heel-forefoot offset $(4 \mathrm{~mm})$ and lacks any motion control or stability features. Most importantly, this shoe is marketed to clinicians and consumers as a minimalist shoe that can provide a barefoot ride. The lightweight racing flat also has a low heel-forefoot offset $(6 \mathrm{~mm})$ and was included in the protocol to determine if differences in running mechanics exist between a minimalist shoe and a lightweight shoe, a shod condition that has been used by runners in training and competition for many years. The insole was in situ for all shod conditions and all reported heel-forefoot offsets and shoe mass include the insole. The regular shoe, minimalist shoe and racing flat had a mean mass (SD) of 323.0 (63.4), 195.5 (19.3) and 184.2 (19.4) g, respectively. All participants were required to complete a 10-day familiarisation period prior to testing. Participants were given the two experimental shod conditions 10 days prior to testing and instructed to complete three runs (minimum) in each of the four conditions prior to testing. Barefoot running familiarisation was conducted on an outdoor athletic track and participants were free to run on any surface during the familiarisation to shod conditions. Distance run during the familiarisation period was collected through a training diary.

\section{Instrumentation}

Running trials were conducted on a $110 \mathrm{~m}$ indoor synthetic running track in the Biomechanics Laboratory of the Australian Institute of Sport. Three-dimensional marker trajectories were captured using a 22-camera VICON motion analysis system (Oxford Metrics Ltd, Oxford, UK), sampling at $250 \mathrm{~Hz}$. Ground reaction force (GRF) data were collected using eight $900 \mathrm{~mm} \times 600 \mathrm{~mm}$ Kistler force plates (Kistler, Amherst, New York, USA), sampling at $1500 \mathrm{~Hz}$. The capture volume had a length of $20 \mathrm{~m}$ and was positioned on the track to allow adequate distance for acceleration and deceleration. Running speed was recorded using timing gates (SpeedLight V2 timing gates, Swift Performance Equipment, Queensland, Australia) positioned at each end of the capture volume.

\section{Procedures}

Participants performed 10 overground running trials in each condition in randomised order. All trials were performed at $90 \%$ of the participant's best $10 \mathrm{~km}$ time in the previous 12 months. Trials were accepted if the velocity was within $\pm 5 \%$ of the target speed. Each participant performed a standardised warm-up that involved five overground running trials within the capture volume. The University of Western Australia's lower body model was used to calculate three-dimensional kinematic and kinetic data. ${ }^{14}$ Data were collected from the right lower limb. Markers were placed on the right and left anterior superior iliac spines and posterior superior iliac spines. Thigh and leg segments were defined by two three-marker clusters. The first cluster was affixed to the lateral aspect of the thigh, aligned with the head of the femur and lateral femoral condyle and the second was affixed to the lateral aspect of the leg, aligned with the lateral femoral condyle and lateral malleolus. The foot was defined by three single retroflective markers fixed to the superoposterior aspect of the calcaneus, and first and fifth metatarsals. In addition, to identify knee-joint and ankle-joint centres, respectively, single retroflective markers were placed on the right medial and lateral femoral condyles and medial and lateral malleoli. The medial markers were removed during the dynamic trials. Marker trajectory data were filtered using a low-pass fourth-order Butterworth filter with a cut-off frequency of $20 \mathrm{~Hz}$. Analogue data were filtered using a low-pass fourthorder Butterworth filter with a cut-off frequency of $50 \mathrm{~Hz}$ to calculate kinetic data. The best cut-off frequencies were determined by performing a residual analyses and visual inspection of the resulting kinematic, kinetic and GRF data.

For each trial, one complete stride cycle was analysed. Joint kinetics were calculated from filtered data using a standard Newton-Euler inverse-dynamics approach; with both male and female segmental inertial properties taken from de Leva. ${ }^{15}$ Three-dimensional joint moments were expressed as internal moments normalised to the product of body mass $(\mathrm{kg})$ and height $(\mathrm{m}){ }^{16}$ Net powers were calculated as a product of the net joint moment and corresponding joint angular velocity. The positive and negative work done during stance was computed for the knee and ankle joints by integrating the relevant portion of the power versus time curve. Both power and work were normalised to body mass $(\mathrm{kg})$. All calculations were performed using VICON Nexus and BodyBuilder software (VICON, Oxford Metrics Ltd.).

\section{Data analysis}

Temporospatial stride characteristics, joint kinematics, moments, power and work were extracted for statistical analysis using a customised MATLAB (Mathworks Inc, Natick, USA) programme. The data for each participant were averaged over the 10 trials for each condition, normalised to the stride cycle $(0-$ $100 \%)$ and graphed over the stance phase. Variables of interest included stride length and stride frequency, lower limb joint angles at footstrike, the maxima and minima of the kinematics, joint moment and power profiles and the positive and negative work done at the knee and ankle joints. Differences between conditions were examined using a multivariate analysis of variance for repeated measures. Mauchley's test of sphericity was conducted to determine if any of the data violated the 
assumption of sphericitiy; in which case the F-ratio and degrees of freedom were taken from the Greenhouse-Geisser epsilon. Post hoc analyses of significant effects were evaluated with univariate F-statistics and Bonferroni correction. The $\alpha$ level was set at 0.05 . For significant post hoc findings the mean difference, 95\% CIs and standardised mean difference (SMD) were calculated. SMD were calculated to express the magnitude of differences between conditions and interpreted according to the following criteria; $\mathrm{SMD} \leq 0.2$, small change; $\mathrm{SMD}=0.5$, moderate change; $\mathrm{SMD}=0.8$ and large change. ${ }^{17}$

\section{RESULTS}

The mean (SD) running velocity across all conditions was 4.48 (1.6) $\mathrm{m} / \mathrm{s}$ and as per the study design there was no difference in running velocity between conditions. Stride length was shorter (range of mean differences: $0.06-0.1 \mathrm{~m}$ ) and stride frequency higher (range of mean differences: 3.8-6.4 steps/min) when running barefoot compared with all conditions (table 2; $\mathrm{p} \leq 0.001)$. Stride length was shorter and stride frequency higher when running in the minimalist shoe and racing flats compared with the regular shoe (table 2), but there were no differences in stride length or frequency between the minimalist and racing flats.

There were significant differences between conditions for kinematic and kinetic variables at the ankle and knee (table 2), with no differences more proximally at the hip. For most variables post hoc testing revealed differences between barefoot and all shod conditions without any differences between the shod conditions. These differences showed moderate to large effects. A graphical representation of the lower extremity joint kinematics and moments related to the gait cycle are shown in figures 1 and 2 .
Peak knee flexion during midstance was decreased (range of mean differences: $2.1-2.4^{\circ}$ ) compared with all shod conditions $(\mathrm{p} \leq 0.001)$, with no differences between shod conditions (figure 1; table 2). This coincided with decreased peak knee extension moments during barefoot running compared with shod. The magnitude of this reduction was $11.9 \%$ (95\% CI $7.3 \%$ to $16.6 \%, \mathrm{p}=0.000, \mathrm{SMD}=0.9), 9.1 \%(95 \%$ CI $5.3 \%$ to $12.9 \%$, $\mathrm{p}=0.000, \mathrm{SMD}=0.7)$ and $11.1 \%(95 \%$ CI $5.8 \%$ to $16.4 \%$, $\mathrm{p}=0.000, \mathrm{SMD}=0.9)$ compared with the minimalist, racing flat and regular shoe, respectively. There was also a $12.6 \%$ $(\mathrm{p}=0.01, \mathrm{SMD}=0.4), 8.4 \%(\mathrm{p}=0.01, \mathrm{SMD}=0.3)$ and $14.2 \%$ $(p=0.04, S M D=0.5)$ reduction in the peak knee abduction moment when running barefoot compared with the minimalist, racing flat and regular shoe, respectively.

When running barefoot the ankle joint was less dorsiflexed (range of mean differences: $3.5-4.5^{\circ}$ ) at initial contact and more plantarflexed (range of mean differences: 4.9-6.1 ${ }^{\circ}$ ) at toe-off compared with all shod conditions $(\mathrm{p} \leq 0.001$, figure 1 and table 2). Peak ankle dorsiflexion and adduction during stance was also reduced when barefoot and in the minimalist shoe compared to the racing flat and regular shoe $(\mathrm{p}=<0.005$ for dorsiflexion and $\mathrm{p} \leq 0.008$ for adduction, table 2 ). The peak ankle plantarflexion moment was $6.1 \% \quad(95 \%$ CI $1.2 \%$ to $11 \%, \quad \mathrm{p}=0.009$, $\mathrm{SMD}=0.5), \quad 8.6 \% \quad(95 \% \quad \mathrm{CI} \quad 2.9 \%$ to $14.2 \%, \mathrm{p}=0.002$, $\mathrm{SMD}=0.7)$ and $7.2 \%(95 \% \mathrm{CI} 0.1 \%$ to $14.2 \%, \mathrm{p}=0.045$, $\mathrm{SMD}=0.5)$ greater when barefoot compared with the minimalist, racing flat and regular shoes, respectively (table 2). Peak ankle inversion moments were greater barefoot compared with the minimalist $(p=0.03, S M D=0.3)$ and racing flat $(p=0.02$, $\mathrm{SMD}=0.5$ ) shoes but not the regular shoe (table 2 ).

Peak power generation was reduced at the knee when barefoot compared with the minimalist and racing flat shoes

Table 2 Group mean (SD) temporospatial, kinematic and kinetic parameters for which repeated-measures MANOVA showed differences $(p<0.05)$ between conditions

\begin{tabular}{|c|c|c|c|c|}
\hline & Barefoot & Minimalist & Racing flat & Regular \\
\hline Stride length (m) & $2.94(0.29)$ & $3.00(0.29)^{*}$ & $3.01(0.30)^{*}$ & $3.04(0.30)^{*, * *, * * *}$ \\
\hline Stride frequency (steps/min) & $187.74(9.49)$ & $183.90(7.8)^{*}$ & $183.90(8.6)^{\star}$ & $181.30(8.1)^{*, * *, * * *}$ \\
\hline \multicolumn{5}{|l|}{ Joint angle (degree) } \\
\hline Peak stance knee flex & $48.57(3.4)$ & $50.67(3.7)^{*}$ & $50.67(3.8)^{*}$ & $50.97(3.6)^{*}$ \\
\hline Ankle contact angle & $0.78(8.4)$ & $4.52(8.1)^{*}$ & $4.25(8.5)^{*}$ & $5.31(8.4)^{*}$ \\
\hline Peak stance ankle dorsifex & $24.94(2.6)$ & $26.09(3.1)$ & $26.33(2.9)^{*}$ & $27.51(2.7)^{*}$ \\
\hline Ankle plantarflex toe-off & $-10.91(9.6)$ & $-6.01(8.4)^{*}$ & $-4.77(9.5)^{*}$ & $-5.09(9.3)^{*}$ \\
\hline Peak stance ankle add & $-9.70(2.5)$ & $-9.37(2.8)$ & $-11.03(3.3)^{*, * *}$ & $-12.55(3.0)^{*, * *}$ \\
\hline \multicolumn{5}{|l|}{ Joint moment $(\mathrm{Nm} / \mathrm{kg} / \mathrm{m})$} \\
\hline Knee ext & $1.72(0.2)$ & $1.92(0.2)^{*}$ & $1.87(0.2)^{*}$ & $1.91(0.2)^{*}$ \\
\hline Knee abd & $0.71(0.2)$ & $0.80(0.2)^{*}$ & $0.77(0.2)^{*}$ & $0.81(0.2)^{*}$ \\
\hline Ankle plantarflex & $1.85(0.2)$ & $1.74(0.2)^{*}$ & $1.69(0.2)^{*}$ & $1.72(0.3)^{*}$ \\
\hline Ankle inversion & $0.41(0.2)$ & $0.35(0.2)^{*}$ & $0.33(0.2)^{*}$ & $0.34(0.2)$ \\
\hline Ankle internal rot & $-0.13(0.1)$ & $-0.10(0.1)$ & $-0.07(0.03)^{*, * *}$ & $-0.05(0.03)^{*, * *}$ \\
\hline \multicolumn{5}{|l|}{ Joint power (W/kg) } \\
\hline Knee power generation & $10.10(2.5)$ & $11.20(3.0)^{*}$ & $11.03(2.9)^{*}$ & $11.13(3.2)$ \\
\hline Ankle power generation & $19.70(3.6)$ & $17.03(3.1)^{*}$ & $16.63(3.4)^{*}$ & $16.98(3.1)^{*}$ \\
\hline Ankle power absorption & $-12.18(3.6)$ & $-10.96(3.0)^{*}$ & $-11.18(2.8)^{*}$ & $-11.75(3.4)$ \\
\hline \multicolumn{5}{|l|}{ Joint work $(\mathrm{J} / \mathrm{kg})$} \\
\hline Knee negative work & $-0.63(0.1)$ & $-0.75(0.2)^{*}$ & $-0.75(0.2)^{*}$ & $-0.78(0.2)^{*}$ \\
\hline Ankle positive work & $1.04(0.2)$ & $0.87(0.1)^{*}$ & $0.84(0.2)^{*, \star *}$ & $0.84(0.2)^{*}$ \\
\hline
\end{tabular}



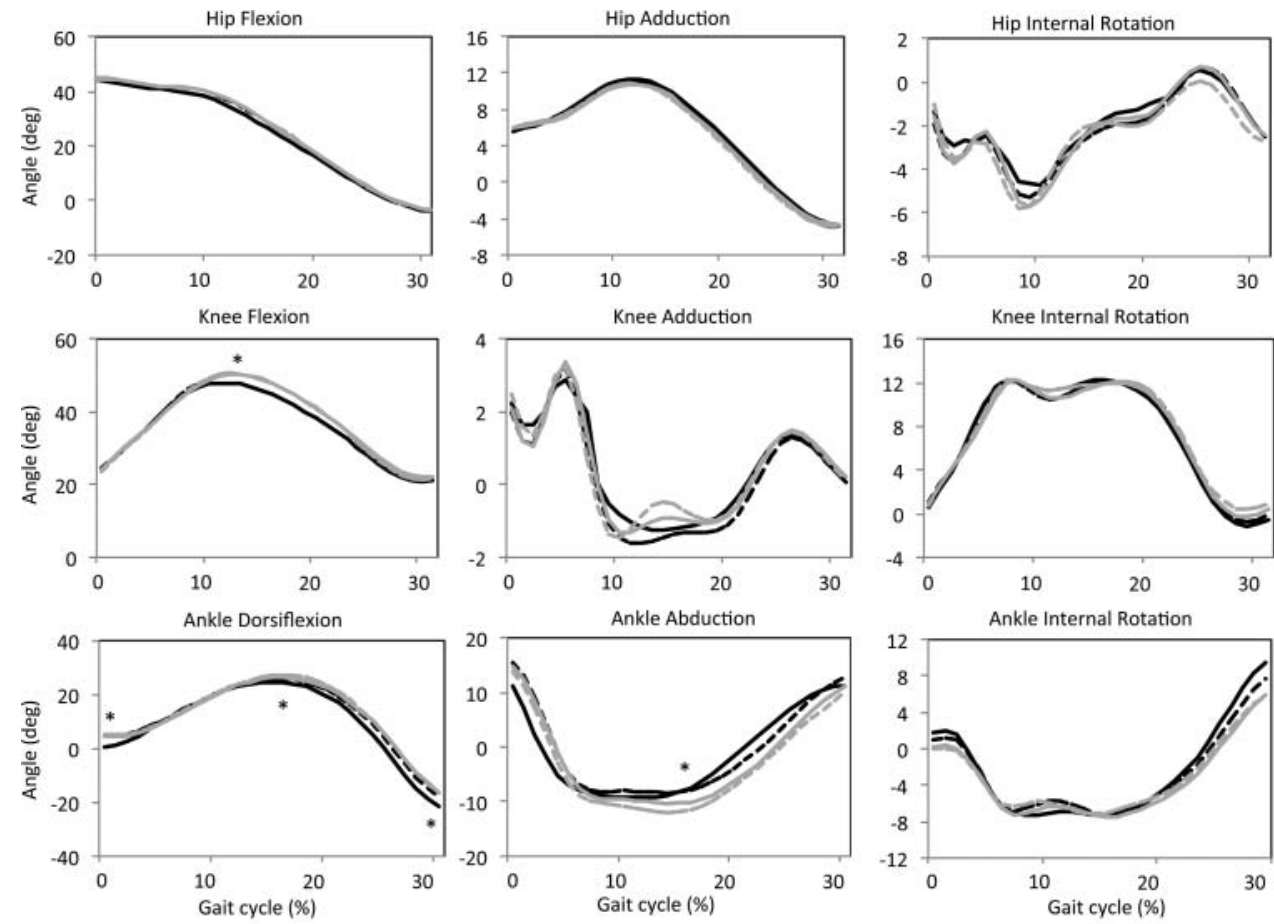

- Barefoot

--- Minimalist $=$ Racing Flat

n=m-Regular

Figure 1 Group mean three-dimensional kinematics at the hip (top panels), knee (middle panels) and ankle (bottoms panels) joints for the stance phase of the gait cycle. Positive values indicate flexion, adduction (abduction at ankle) and internal rotation. Negative values indicate extension, abduction (adduction at ankle) and external rotation. *Indicates significant difference between conditions for joint angle at contact and peak joint angle.

$(10.9 \%, p=0.04, \mathrm{SMD}=0.4$ and $9.2 \%, \mathrm{p}=0.04, \mathrm{SMD}=0.3$, respectively), while an increase in peak power generation at the ankle was seen when barefoot compared with all shod conditions $(13.6-15.6 \%, \mathrm{p} \leq 0.001, \mathrm{SMD}=0.8-0.9$; table 2$)$. Peak power absorption at the ankle was also greater when barefoot compared with the minimalist and racing flat shoes
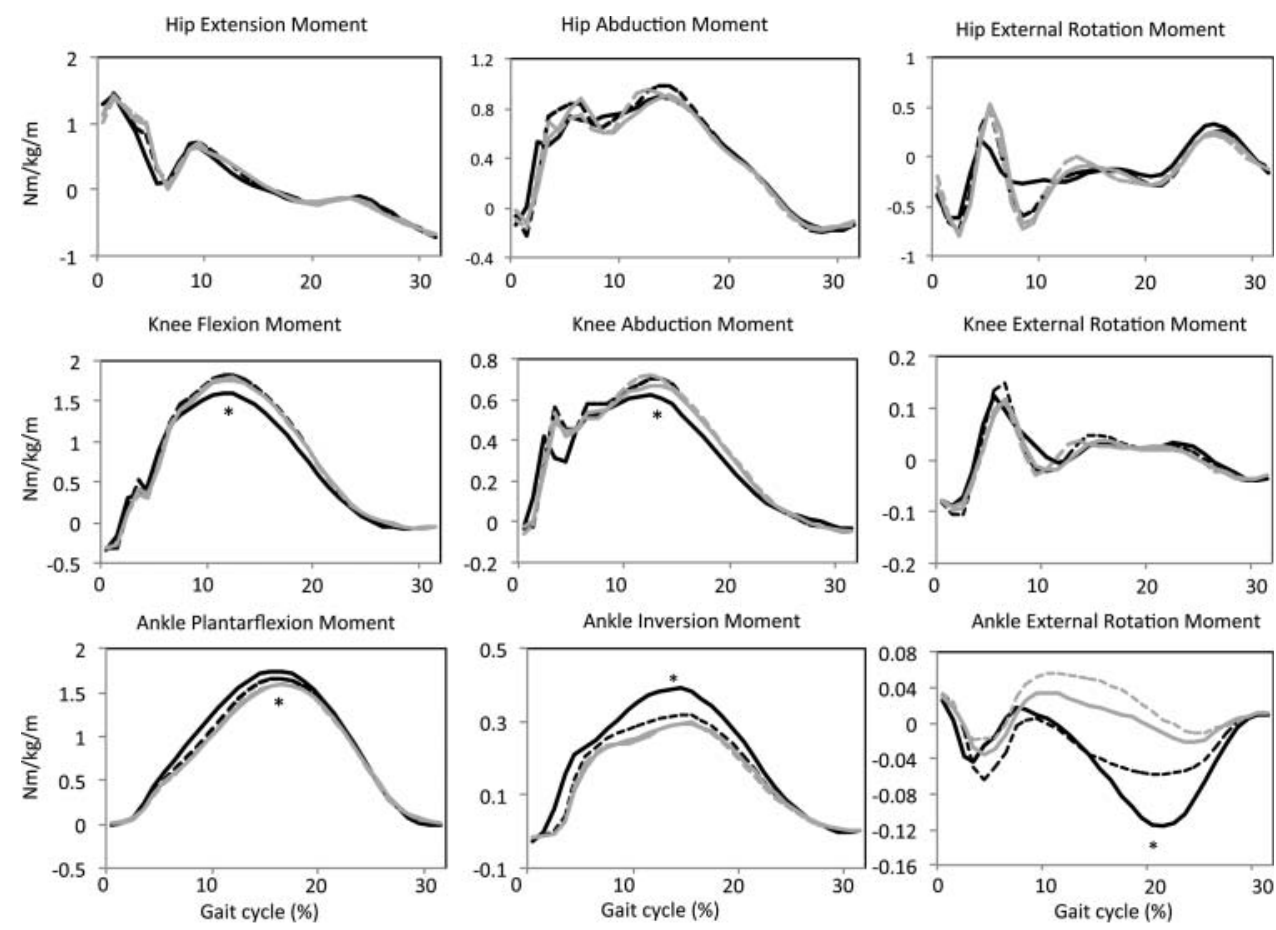

Barefoot

---- Minimalist $=$ Racing Flat

nems Regular

Figure 2 Group mean three-dimensional moments at the hip (top panels), knee (middle panels) and ankle (bottoms panels) joints for the stance phase of the gait cycle. Positive values indicate extension, abduction and external rotation. Negative values indicate flexion, adduction and internal rotation. *Indicates significant difference between conditions for peak joint moments. 
$(\mathrm{p}=0.03$ and 0.02 , respectively, table 2$)$. While barefoot there was a $19.7 \%(95 \%$ CI $9.7 \%$ to $29.7 \%, p=0.000, S M D=0.8)$, $18.9 \%(95 \%$ CI $10 \%$ to $27.8 \%, \mathrm{p}=0.000, \mathrm{SMD}=0.7)$ and $23.7 \%(95 \%$ CI $13.8 \%$ to $33.5 \%, \mathrm{p}=0.000, \mathrm{SMD}=0.9)$ reduction in the negative work at the knee joint compared with the minimalist, racing flat and regular shoe, respectively (table 2). Conversely, running barefoot increased the positive work at the ankle compared with all shod conditions. The magnitude of the reduction in negative work at the knee joint was similar to the magnitude of the increase in the positive work at the ankle when barefoot compared with shod (16.6-20\%, p $\leq 0.001$, $\mathrm{SMD}=1.0-1.2$; table 2).

\section{DISCUSSION}

The results of this study support our hypothesis that barefoot running induces changes to the running mechanics of highly trained habitual shod runners. In comparison with shod, running barefoot demonstrated a less dorsiflexed ankle at initial contact, a less flexed knee during midstance, lesser joint moments and work done at the knee and greater joint moments and work done at the ankle. Knee and ankle mechanics when running barefoot were different to all shod conditions, including the minimalist shoe, indicating that the minimalist shoe cannot entirely replicate the mechanics of running barefoot. Other than small differences in stride length and stride frequency there was very little difference in running mechanics between shod conditions, suggesting that barefoot running is inherently different to shod running and that different shoes have little impact on highly trained runners' gait. The findings of this study support previous work in recreational athletes that the dynamics of running barefoot are different to shod running in asymptomatic individuals. 358

The only other study to compare barefoot running to running in a minimalist shoe found that the ankle contact angle was similar between barefoot running and running in Vibram Fivefingers. ${ }^{8}$ Both of these conditions displayed a less dorsiflexed ankle at initial contact compared with a standard neutral shoe. Sagittal plane knee kinematics were not different between conditions. Methodological considerations and the type of minimalist shoe used may explain the different findings between studies. The minimalist shoe used in the latter study has a $3.5 \mathrm{~mm}$ rubber sole with limited cushioning whereas the minimalist shoe in the current study has a soft heel of $17 \mathrm{~mm}$ that still affords relatively considerable cushioning. It has been suggested that cushioning (along with an elevated heel) enables runners to land with a dorsiflexed ankle by limiting the discomfort associated with heel contact. ${ }^{5}$ Our findings of a shorter stride length and higher stride frequency during barefoot running is consistent with the findings of others, ${ }^{6} 8$ and aspects of barefoot running form discussed by Lieberman. ${ }^{18}$ Unlike in the barefoot condition, although runners adopted shorter stride lengths when running in the minimalist and racing flat shoes compared with the regular shoe, they did not alter their ankle angle at contact. Perhaps the cushioning, elevated heel and lack of proprioception afforded by the shoes used in our study still encourages runners to adopt a rearfoot footstrike pattern.

We did not find any kinematic or kinetic differences between the minimalist shoe and the other shod conditions, which is in contrast to the findings of others. ${ }^{8}$ The regular shoe used in our study was not controlled to a neutral or stability-type construction. It is possible that by not controlling the regular shoe, differences between the minimalist and a neutral or stability shoe were overlooked. Of note is that in contrast to other studies our participants were highly trained runners. It may be proposed that athletes at that level already have highly consistent running mechanics ${ }^{19} 20$ and different types of shoes have little influence on their running gait. It is possible that lesser trained runners with less consistent mechanics ${ }^{19}{ }^{20}$ may be more susceptible to changes in running gait when utilising a minimalist shoe.

The less dorsiflexed ankle at initial contact when barefoot running is consistent with previous findings. ${ }^{3}{ }^{8}$ Less dorsiflexion reduces the pressure under the heel ${ }^{8}$ and may be an attempt to reduce the discomfort associated with the large and rapid impact peak that occurs when rearfoot striking barefoot. ${ }^{5}$ We also observed smaller knee flexion angles at midstance when running barefoot but no differences between conditions for the knee flexion angle at contact which is consistent with previous reports. ${ }^{7821}$ Stacoff et $a l^{22}$ used in vivo methods (bone pins) to show that three-dimensional tibiocalcaneal rotations did not differ substantially between barefoot and shod running. They also suggested that skin-based or shoe-based marker systems overestimated movement and this artefact may be reflected in other such studies. While we acknowledge the limitation of measuring kinematics of the ankle by assuming the foot as a rigid segment and placing markers on the shoe itself, besides ankle contact and toe-off angles, the kinematic differences were small $\left(\mathrm{ie},<3^{\circ}\right.$ ). The clinical importance of these small changes is unknown with kinetic or internal loads potentially of greater relevance.

The reduced knee flexion during barefoot running was associated with a $9-12 \%$ decrease in the knee extension and abduction moments compared to the shod conditions. Kerrigan et al found a much larger increase (36-38\%) in knee joint moments when running on a treadmill in a neutral shoe compared with barefoot. The differences may be explained by the different shoes utilised, the mode of testing (treadmill vs overground) or differences in running kinematics; although Kerrigan et $a l^{6}$ did not report kinematics. Nonetheless larger knee extension and abduction joint moments during shod running may have implications for knee pain and injury. The smaller knee flexion angle during barefoot running reduces the moment arm and the resultant knee extension moment; which can reduce the stress across the patellofemoral joint. ${ }^{23}$ However, we acknowledge that the methods employed in this study cannot determine the actual joint contact forces.

Similar to the findings of Perl et $a l^{7}$ we found an increase in the ankle plantarflexion moment during barefoot running. This increase implies an increase in the work of the triceps surae muscles. Unlike others we also calculated net joint powers and work done; and of all the variables calculated it is work done that conveys the most important information regarding muscle function at the lower-limb joints. ${ }^{24}$ Our results show a $19-24 \%$ decrease in negative work done at the knee and a $13-15 \%$ increase in peak power generation and $16-19 \%$ increase in positive work done at the ankle during barefoot running compared with the shod conditions. This increase in work of the triceps surae muscles during barefoot running may account for the large majority of anecdotal reports of calf and Achilles soreness when first undertaking barefoot running. The long-term implication of this increase in load through the triceps surae requires further investigation. Conversely, the decrease in work at the knee during barefoot running may have therapeutic benefits for runners with knee pain and injury. At present there are only anecdotal reports of injury incidence in barefoot populations ${ }^{1}$ and controlled prospective studies are required.

There are limitations to this study that must be considered. First, we studied the impact of running barefoot on habitually shod runners' gait. We do not propose that our results are 


\section{What this study adds}

- Shoes marketed as minimalist may not replicate the mechanics of running barefoot.

- Compared with a runner's regular shoe, minimalist and lightweight shoes have only a small impact on highly trained runners gait.

\section{How might it impact on clinical practice in the near} future

- Prescription of minimalist shoes as a mechanism to change running mechanics may not be justified.

- Barefoot running increases in work at the ankle and reductions in work at the knee should be considered in training and rehabilitation.

reflective of the running mechanics of habitual barefoot populations, as others have shown that differences in barefoot running mechanics exist between these populations. ${ }^{5}$ Second, the minimalist shoe used in the current study has cushioning and an elevated heel therefore care must be taken when extrapolating our results to different types of minimalist shoes, especially those without cushioning or an elevated heel. Finally, our participants were asymptomatic so care must be taken when extrapolating our results to symptomatic populations as these individuals may respond differently to barefoot and shod conditions.

In conclusion, the dynamics of running overground while barefoot are different to that of running in a minimalist shoe that has cushioning and an elevated heel. Athletes and their coaches should not expect to instantly replicate barefoot running while in a minimalist shoe. Running barefoot does induce mechanical changes to habitually shod highly trained runners gait and it is inherently different to shod running. The increase in work done at the ankle must be considered when transitioning to running barefoot as too rapid a transition may overload the triceps surae complex. Conversely, the reduction in joint moments and work done at the knee while running barefoot may provide potential benefits for the management of knee pain and injury.

Acknowledgements We are grateful to the School of Sport Science, Exercise and Health at the University of Western Australia for use of the lower limb kinematic and kinetic model. We thank NIKE for providing the minimalist and lightweight racing flat footwear.

Contributors JB was involved in the concept and design of the study, conducted the data collection and analysis and prepared the manuscript. PUS recruited the participants and was involved in preparation of the manuscript. AH assisted with data collection and analysis and manuscript preparation. TR was involved in the data analysis and interpretation. BTV was involved in the concept and design of the study, data analysis and interpretation and preparation of the manuscript.

WS conducted the data collection and was involved in interpretation of the data and preparation of the manuscript.

Competing interests None.

Ethics approval Deakin University and Australian Institute of Sport Human Ethics Committee.

Provenance and peer review Not commissioned; externally peer reviewed.

\section{REFERENCES}

1 Robbins S, Hanna A. Running-related injury prevention through barefoot adaptations. Med Sci Sports Exerc 1987;19:148-56.

2 De Wit B, De Clercq D. Timing of lower extremity motions during barefoot and shod running at three velocities. J App/ Biomech 2000;16:169-79.

3 De Wit B, De Clercq D, Aerts P. Biomechanical analysis of the stance phase during barefoot and shod running. J Biomech 2000;33:269-78.

4 Divert $\mathrm{C}$, Baur $\mathrm{H}$, Mornieux $\mathrm{G}$, et al. Stiffness adaptations in shod running. J Appl Biomech 2005;21:311-21.

5 Lieberman DE, Venkadesan M, Werbel WA, et al. Foot strike patterns and collision forces in habitually barefoot versus shod runners. Nature 2010;463:531-5.

6 Kerrigan C, Franz JR, Keenan G, et al. The effect of running shoes on lower extremity joint torques. Phys Med Rehabil 2009;1:1058-63.

7 Perl D, Daoud A, Lieberman D. Effects of footwear and strike type on running economy. Med Sci Sports Exerc 2012;44:1335-43.

8 Squadrone R, Gallozzi C. Biomechanical and physiological comparison of barefoot and two shod conditions in experienced barefoot runners. I Sports Med Phys Fitness 2009;49:6-13.

9 Nigg B, De Boer R, Fisher V. A kinematic comparison of overground and treadmill running. Med Sci Sports Exerc 1995;27:98-105.

10 Riley PO, Dicharry J, Croce UD, et al. A kinematics and kinetic comparison of overground and treadmill running. Med Sci Sports Exerc 2008;40:1093-100.

11 Noehren B, Davis I, Hamill J. Prospective study of the biomechanical factors associated with iliotibial band syndrome. Clin Biomech 2007;22:951-6.

12 Willems TM, De Clercq D, Delbaere K, et al. A prospective study of gait related risk factors for exercise-related lower leg pain. Gait Posture 2006;23:91-8.

13 Willems TM, Witvrouw E, De Cock A, et al. Gait-related risk factors for exercise-related lower-leg pain during shod running. Med Sci Sports Exerc 2007;39:330-9.

14 Besier TF, Sturnieks DL, Alderson JA, et al. Repeatability of gait data using a functional hip joint centre and a mean helical knee axis. J Biomech 2003;36:1159-68.

15 de Lava P. Adjustments to Zatsiorsky-Seluyanov's segment inertia parameters. J Biomech 1996;29:1223-30.

16 Moisio KC, Sumner DR, Shott $S$, et al. Normalization of joint moments during gait: a comparison of two techniques. J Biomech 2003;36:599-603.

17 Cohen J. Statistical power analysis for the behavioral sciences. 2nd edn. Hillsdale, NJ: Lawrence Erlbaum Associates, 1988:40.

18 Lieberman DE. What can we learn about running from barefoot running: an evolutionary perspective. Exerc Sport Sci Rev 2012;40:63-72.

19 Chapman A, Vicenzino B, Blanch $\mathrm{P}$, et al. Is running less skilled in triathletes than runners matched for running training history. Med Sci Sports Exerc 2008;40:557-65.

20 Hreljac A. Stride smoothness evaluation of runners and other athletes. Gait Posture 2000;11:199-206.

21 Braunstein $B$, Arampatzis $A$, Eysel $P$, et al. Footwear affects the gearing at the ankle and knee joints during running. J Biomech 2010;43:2120-5.

22 Stacoff A, Nigg BM, Reinschmidt C, et al. Tibiocalcaneal kinematics of barefoot versus shod running. J Biomech 2000;33:1387-95.

23 Reilly DT, Martens M. Experimental analysis of quadriceps muscle force and patello-femoral joint reaction force for various activities. Acta Orthop Scand 1972;43:126-37.

24 Winter DA. Biomechanics and motor control of human movement. New York: John Wiley \& Sons, Inc, 2009:140. 